\title{
Opposition-Based Improved PSO for Optimal Reactive Power Dispatch and Voltage Control
}

\author{
Shengrang Cao, ${ }^{1,2}$ Xiaoqun Ding, ${ }^{1}$ Qingyan Wang, ${ }^{1}$ and Bingyan Chen ${ }^{1,3,4}$ \\ ${ }^{1}$ College of Energy and Electrical Engineering, Hohai University, Nanjing 211100, China \\ ${ }^{2}$ Jiangsu Union Technical Institute, Nanjing Branch, Nanjing 210019, China \\ ${ }^{3}$ Hohai University, Nantong Institute of Marine and Offshore Engineering, Nantong 226000, China \\ ${ }^{4} J i a n g s u$ Province Key Laboratory of Environmental Engineering, Nanjing 210000, China \\ Correspondence should be addressed to Shengrang Cao; csrhhu@foxmail.com
}

Received 11 September 2014; Revised 31 December 2014; Accepted 3 March 2015

Academic Editor: Pandian Vasant

Copyright (C) 2015 Shengrang Cao et al. This is an open access article distributed under the Creative Commons Attribution License, which permits unrestricted use, distribution, and reproduction in any medium, provided the original work is properly cited.

\begin{abstract}
An opposition-based improved particle swarm optimization algorithm (OIPSO) is presented for solving multiobjective reactive power optimization problem. OIPSO uses the opposition learning to improve search efficiency, adopts inertia weight factors to balance global and local exploration, and takes crossover and mutation and neighborhood model strategy to enhance population diversity. Then, a new multiobjective model is built, which includes system network loss, voltage dissatisfaction, and switching operation. Based on the market cost prices, objective functions are converted to least-cost model. In modeling process, switching operation cost is described according to the life cycle cost of transformer, and voltage dissatisfaction penalty is developed considering different voltage quality requirements of customers. The experiment is done on the new mathematical model. Through the simulation of IEEE 30-, 118-bus power systems, the results prove that OIPSO is more efficient to solve reactive power optimization problems and the model is more accurate to reflect the real power system operation.
\end{abstract}

\section{Introduction}

Particle swarm optimization (PSO) proposed by Dr. Eberhart and Dr. Kennedy in 1995 is a population-based evolutionary algorithm that emulates the social behavior of bird flocks in an attempt to optimally explore some given problem space. In the past decade, PSO has been studied and applied in many research and application areas. However, many experiments have shown that PSO converges too fast and easily falls into local optima especially when solving complex multimodal problems [1]. Many researchers have put forward improved programs. In general, there are three kinds of improved methods, that is, parameter factor [2, 3], neighborhood topology [4], and hybridization with other algorithms [5, 6]. Each of them has its own advantages but also has defects. The hybridization of PSO with other algorithms has been proved to be a promising technique. There are two ways to combine. One is with evolutionary algorithms, for instance, [5] is PSO combined with genetic algorithm, in which genetic algorithm was used to increase exploration ability of particles, [6] is the combination hybridized PSO with ant algorithm, and uses the characteristic of positive feedback to enhance the search ability of particles. The other is with nonevolutionary algorithms, such as hybridizing PSO with Filter [7], Cauchy mutation [8], and opposition-based learning [9], in which opposition-based learning has a good character; it can accelerate the convergence speed of the algorithm, and this concept was first proposed by Dr. Tizhoosh. When we seek the solution in a direction, it is beneficial to consider the opposite direction [10]. By comparison of fitness values of the two directions, the optimal candidate solution is chosen from them. For this reason, many algorithms were enhanced by opposition-based learning $[11,12]$.

In this paper, a new improved particle swarm optimization algorithm OIPSO is proposed, which makes some expansion and correction by inertia weight factors, crossover and mutation, neighborhood model, and opposition-based learning. Through these strategies, the new OIPSO algorithm can enhance population diversity and avoid premature convergence and stagnation. 
The improved algorithm would be developed to multiobjective reactive power optimization. It is known that the purpose of power system reactive optimization is to find the best compensation methods to make the power system safe and economic under the demand of reactive load power system [13]. For that, we establish a mathematical model, including system network loss, voltage dissatisfaction, and switching operator. In the modeling process, introduce life cycle cost of the transformer, define switching operator cost, and also give descriptions of the customers' loss, voltage quality requirement, and dissatisfaction degree and then reactive power optimization tests through IEEE 30- and 118bus systems.

The remaining of this paper is organized as follows: Section 2 provides an overview of PSO algorithm, opposition-based learning, and other improvement strategies. In Section 3, the multiobjective model of reactive power system is established. Combined with reactive power optimization, main steps of the improved OIPSO algorithm are given in Section 4. Then, the algorithm is applied to IEEE 30- and 118-bus systems in Section 5. Finally, Section 6 concludes the paper with a summary.

\section{PSO Algorithm and Its Improvement}

The essence of PSO algorithm is that by letting the information about good solutions spread out through the swarm, the particles would tend to move to good areas $[14,15]$. At each iteration time $t$, particle $i$ is moved to a new position by adding a velocity term to its current position according to formulas (1) and (2) as follows:

$$
\begin{gathered}
X_{i}(t+1)=X_{i}(t)+V_{i}(t+1) \\
V_{i}(t+1)=\omega V_{i}(t)+c_{1} \operatorname{rand}_{1}\left(P_{\text {best }, i}-X_{i}(t)\right) \\
+c_{2} \operatorname{rand}_{2}\left(G_{\text {best }}-X_{i}(t)\right)
\end{gathered}
$$

where $i=1,2, \ldots$, pop, pop is the size of swarm particles, $P_{\text {best }, i}$ is the best position of particle $i, G_{\text {best }}$ is the global best position, $\omega$ is called inertia weight, and $c_{1}$ and $c_{2}$ are acceleration factors.

PSO algorithm converges fast, but it is also easy to fall into the local optimal. The reason is that, in the optimal process, all particles consider the global best position $G_{\text {best }}$ as the goal and search directions run towards it. This situation worsens in the later process and leads to reduction of the ability to explore unknown area. Therefore, it is very important to enhance the diversity of particles. This paper makes some expansion and modification to the basic particle swarm algorithm. The main improvement measures are as follows.

2.1. Opposition-Based Learning. In PSO algorithm, starting points are given randomly. If the starting points are close to the optimal point, convergence speed would be faster. The opposite operation used in the selection of the starting points has proven that utilizing opposition in learning yields more efficient algorithms than using only pure randomness $[10,16]$. Besides the opposition point used in the initial population, this paper would intersectionally use the opposition point and the following crossover and mutation to increase the diversity of particles. The definition of opposition is given as follows.

Definition 1 (opposite point). Let $X=\left(x_{1}, x_{2}, \ldots, x_{n}\right)$ be a point in $n$-dimensional space and let $x_{i} \in\left[a_{i}, b_{i}\right]$ be a real number, where $i=1,2, \ldots, n$. The opposite point $X^{\prime}=$ $\left(x_{1}^{\prime}, x_{2}^{\prime}, \ldots, x_{n}^{\prime}\right)$ is defined by its components $x_{i}^{\prime}=a_{i}+b_{i}-x_{i}$.

2.2. Inertia Weight Factor. Generally, for initial stage of search process, large inertia weight can enhance the global exploration and, for last stage, the small inertia weight is good for local exploration, so time-varying inertia weight, which typically decreases linearly from about 0.9 to 0.4 [17], can balance the global and local search; specific measures are as follows:

$$
\omega=\omega_{\max }-\frac{t}{t_{\max }}\left(\omega_{\max }-\omega_{\min }\right)
$$

where $\omega_{\max }$ and $\omega_{\min }$ are the maximum and minimum of $\omega, t_{\max }$ is the maximum iterating times, and $t$ is the current iteration.

2.3. Neighborhood Exchange. In particle swarm algorithm, each particle generally learns from its own best position and global best position. In social cognitive system, an individual, besides its own experience and excellent information obtained from the whole society, should exchange with other better individuals to improve itself $[13,18]$. Based on this idea, PSO algorithm is modified as follows:

$$
\begin{aligned}
V_{i}(t+1)= & \omega V_{i}(t)+c_{1} \operatorname{rand}_{1}\left(P_{\text {best }, i}-X_{i}(t)\right) \\
& +c_{2} \operatorname{rand}_{2}\left(G_{\text {best }}-X_{i}(t)\right) \\
& +c_{3} \operatorname{rand}_{3}\left(P_{\text {best }, n}-X_{i}(t)\right)
\end{aligned}
$$

where $P_{\text {best }, n}$ is position vector of the better individual in domain and $c_{3}$ is an accelerating constant. Larger $c_{3}$ can increase the ability of the individual to explore unknown better area, especially in the later process. In contrast, the set value of $c_{2}$ is related to convergence rate; based on their own characteristics, parameters $c_{2}, c_{3}$ are improved as follows:

$$
\begin{aligned}
& c_{3}=c_{3 \min }+\frac{t}{t_{\text {max }}}\left(c_{3 \max }-c_{3 \min }\right), \\
& c_{2}=c_{2 \max }-\frac{t}{t_{\max }}\left(c_{2 \max }-c_{2 \min }\right),
\end{aligned}
$$

where $c_{2 \max }$ and $c_{2 \text { min }}$ are the maximum and minimum of $c_{2}$ and $c_{3 \max }$ and $c_{3 \text { min }}$ are the maximum and minimum of $c_{3}$.

2.4. Crossover and Mutation. Crossover and mutation operators are key technologies in genetic algorithm, which are used to enhance the diversity of the species. The particles of PSO 
algorithm can be crossed and varied $[13,18]$. For particle $i$, the crossover operation is described as follows:

$$
\begin{aligned}
& V_{i}(t+1)=\frac{V_{i}(t)+V_{n}(t)}{\left\|V_{i}(t)+V_{n}(t)\right\|}\left\|V_{i}(t)\right\|, \\
& X_{i}(t+1)=r X_{i}(t)+(1-r) X_{n}(t),
\end{aligned}
$$

where $r$ is a random variable in the range $[0,1]$.

The method of mutation operator is

$$
\begin{aligned}
& V_{i}(t+1)=\frac{V_{i}(t)+V_{i}(t+1)}{\left\|V_{i}(t)+V_{i}(t+1)\right\|}\left\|V_{i}(t)\right\|, \\
& X_{i}(t+1)=\gamma X_{i}(t)+(1-r)\left(X_{i \max }-X_{i \min }\right),
\end{aligned}
$$

where $\gamma$ is a random coefficient in the range $[0,1] . X_{i \max }$, $X_{i \text { min }}$ are the maximum and minimum of $X_{i}$. A variable parameter $P_{m}$ selected from $[0,1]$ determines the mutation operator.

\section{Mathematical Model of Power System Reactive Power Optimization}

Objective function of reactive power optimization model is commonly the minimization of active power loss and ignores many security and economic factors. On security grounds, its optimal results often approach the upper limits of voltage level on load buses. Although the results satisfy equality and inequality constraints, it is not conducive to the safe operation of power system. In this paper, we introduce voltage dissatisfaction and use penalty to punish voltage dissatisfaction. And, in economic terms, transformer tap has mechanical life and the total number of switching times has a limit. Therefore, the switching operation cost should not be neglected. In this section, we present transformer operation cost by life cycle cost and use it as an objective function.

3.1. Active Power Loss. Reactive power is usually used as the objective function. Utilize the following equal to convert power loss into expense:

$$
C_{P \text { loss }}=\lambda_{1} \times P_{\text {loss }}=\lambda_{1} \times \sum_{\substack{i \in N \\ j \in N_{i}}} G_{i j}\left(V_{i}^{2}+V_{j}^{2}-2 V_{i} V_{j} \cos \theta_{i j}\right),
$$

where $\lambda_{1}$ is the unit price for system network loss and its value depends on market electricity prices, $P_{\text {loss }}$ is system network loss, $N$ is set of branches numbers, and $N_{i}$ is the collection of nodes number associated with the node $i$ (including $i$ itself).

3.2. Voltage Dissatisfaction. The cost of voltage dissatisfaction describes the users' losses caused by overlimit voltages on the load buses. When the voltage amplitudes are far from the ideal range, the losses would become more heavy. Voltage dissatisfaction can be expressed by the following mathematical description [19]:

$$
\begin{gathered}
C_{\mathrm{VVIO}}=\lambda_{2} \times S_{V}=\lambda_{2} \times \sum_{i=1}^{N_{\text {load }}} S_{V i}, \\
S_{V i}= \begin{cases}a_{i} \times P_{D i} \times\left(V_{i}^{\min }-V_{i}\right)^{\theta} & V_{i}<V_{i}^{\min } \\
0 & V_{i}^{\min }<V_{i}<V_{i}^{\max } \\
b_{i} \times P_{D i} \times\left(V_{i}-V_{i}^{\max }\right) & V_{i}^{\max }<V_{i},\end{cases}
\end{gathered}
$$

where $\lambda_{2}$ is the penalty for voltage dissatisfaction; $S_{V}$ is the sum of nodal voltage dissatisfaction degree; $P_{D i}$ is the active power load of node $i ; a_{i}, b_{i}$ are the voltage dissatisfaction degree when the voltage values overstep the lower or upper limit, which are declared by the relevant users; $\theta$ is the index parameter about the relationship of the losses and voltage deviation; we set $\theta=1$ in this paper. In general, voltage range is $[0.94,1.06]$, but some power consumption equipment needs high security and stability. It is obvious that the voltages are better to control in a satisfactory interval, instead of approaching upper limits, so we change voltage range into satisfactory interval. The satisfactory interval is set by users at different load buses. In this paper, we set satisfactory interval to be $[0.95,1.05]$ if voltages satisfy equality and inequality constraints but overstep the satisfactory interval, and dissatisfactory penalty can be relatively small and can be set based on users' requirement of electricity quality.

3.3. Switching Operations. Transformer operation cost is mainly composed of construction, installation, equipment acquisition, maintenance, and so on. Define $S_{T i}$ as the change of the ratio at transformer $i$ before and after optimization and it is described as

$$
S_{T i}=\frac{\left|T_{i}-T_{i 0}\right|}{\Delta T_{i}}
$$

where $T_{i}$ is the ratio of transformer $i$ after optimization; $T_{i 0}$ is the ratio of transformer $i$ before optimization; $\Delta T_{i}$ is the step of transformer $i$. After optimization, tap operation costs are

$$
C_{\mathrm{TC}}=\lambda_{3} \times S_{T}=\lambda_{3} \times \sum_{i=1}^{N_{T}} S_{T i}
$$

where $N_{T}$ is the total of transformers and $\lambda_{3}$ is the unit price of each action, which depends on the annual value of investment, operation, and maintenance cost, where each of them is described as follows.

3.3.1. Construction Cost. Construction cost of the transformer is composed of construction project cost, installation project cost, equipment acquisition cost, and other cost, called static investment $[20,21]$. Generally, construction cost is expressed as

$$
C_{1}=V_{1} \cdot \frac{\gamma \cdot(1+\gamma)^{n}}{(1+\gamma)^{n}-1}
$$

where $C_{1}$ is the annual value of the lump-sum investment, $V_{1}$ is the lump-sum investment, and $\gamma$ is the interest rate. 
3.3.2. Scrap Value. Scrap value of the transformer can be divided into residual recovery income, early retirement loss cost, and disposal expenses. Its annual value is described as [19]

$$
C_{2}=-\rho \cdot C_{1}+\frac{\left(n-n^{\prime}\right)}{n} \cdot C_{1}+\mathrm{dc} \cdot \frac{\gamma \cdot(1+\gamma)^{n}}{(1+\gamma)^{n}-1}
$$

$\rho$ is the recovery factor, and we set it as $20 \% \sim 30 \%$. The minus sign expresses that the income is negative relative to the expenditure; dc is the current value of the disposal expense; $n$ is the actual operating life; $n^{\prime}$ is the expected operating life. When the actual operating life is greater than or equal to the expected life, early retirement loss cost is 0 .

3.3.3. Maintenance Cost. The maintenance method adopts scheduling repair; its model is described as

$$
V_{3}=\sum_{t=1}^{30} M_{e} \cdot\left(1-\exp \left(-\left(\frac{t}{15.98}\right)^{1.35}\right)\right),
$$

where $M_{e}$ is early operation and maintenance cost; $t$ is the year number; and $V_{3}$ is the total cost of maintenance in 30 years. Use the uniform annual value method for conversion; maintenance cost of each year can be expressed as

$$
C_{3}=V_{3} \cdot \frac{\gamma \cdot(1+\gamma)^{n}}{(1+\gamma)^{n}-1} .
$$

Through the above models, the life cycle cost (LLC) is defined as

$$
\mathrm{LCC}=\mathrm{C}_{1}+\mathrm{C}_{2}+\mathrm{C}_{3} .
$$

Take the $110 \mathrm{kv}$ conventional substation [18]. For example, set the expected life of the transformer $n^{\prime}$ to be 30 years. $\gamma$ is $8 \%$, the mechanical life of tap is 10000 times, and average annual switching operations are 333 times. For transformer tap, its unit price of one action in the $t$ th year is

$$
C_{t_{\_a v g}}=\frac{C_{1}+C_{2}+C_{3}}{333} .
$$

In this paper, we set $\lambda_{3}=C_{1 \_ \text {avg }}$.

3.4. Objective Function and Constraints. To satisfy the cost savings and security purposes, this paper combines system network loss, voltage dissatisfaction, and switching operation as the optimization objective. Voltage dissatisfaction can be converted into economic cost, so the objective function can be expressed as

$$
\min C_{\text {tot }}=C_{\text {Ploss }}+C_{\mathrm{VVIO}}+C_{\mathrm{TC}} .
$$

The function should satisfy the equality and inequality constraints, where equality constraints reflect the physics of the power system shown as

$$
\begin{gathered}
P_{G i}-P_{L i}=V_{i} \sum_{j=1}^{N} V_{j}\left(G_{i j} \cos \delta_{i j}+B_{i j} \sin \delta_{i j}\right), \\
Q_{G i}-Q_{L i}+Q_{C i}=V_{i} \sum_{j=1}^{N} V_{j}\left(G_{i j} \sin \delta_{i j}-B_{i j} \cos \delta_{i j}\right),
\end{gathered}
$$

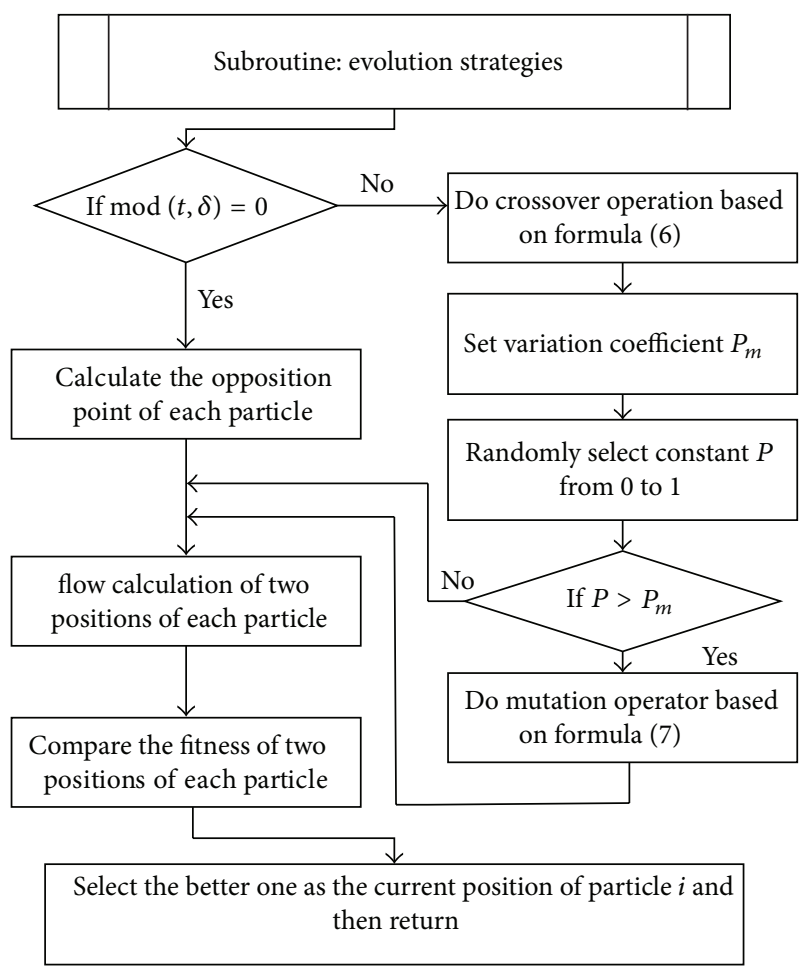

FIGURE 1: Subroutine of evolution strategies.

where $N$ is node number; $P_{G i}$ and $P_{L i}$ are generator active power output and active power of load at node $i$; and $Q_{G i}$, $Q_{L i}$, and $Q_{C i}$ are generator reactive power input, load reactive power, and reactive power of the compensator capacitor at node $i$.

Inequality constraints are about the limits of active power, reactive power, voltage, capacitance, and the times of switching operations and are created to ensure system security [12] expressed as

$$
\begin{array}{cc}
P_{G i \min } \leq P_{G i} \leq P_{G i \max } \quad\left(i \in N_{G}\right), \\
Q_{G i \min } \leq Q_{G i} \leq Q_{G i \max } \quad\left(i \in N_{G}\right), \\
V_{i \min } \leq V_{i} \leq V_{i \max } \quad(i \in N), \\
Q_{C i \min } \leq Q_{C i} \leq Q_{C i \max } \quad\left(i \in N_{C}\right), \\
T_{i \min } \leq T_{i} \leq T_{i \max } \quad\left(i \in N_{T}\right),
\end{array}
$$

where $N_{G}$ is the set of generation units; $N_{T}$ is the set of tap transformers; and $N_{C}$ is the set of compensator capacitors.

\section{Reactive Power Optimization Using the Opposition-Based Improved PSO}

Crossover and mutation operation and opposition-based learning are two improvement strategies. We combine and apply them to enhance diversity of particles. The specific process is shown in Figure 1. The main steps are as follows.

Step 1. Define the input data. The input data include the generator voltages and the transformer tap settings, reactive 
power of switchable VAR sources, the population size of the particle swarm, the maximum number of iterations, and accelerated constants.

Step 2. Initialize the population. The initial population is generated randomly, which must meet the constraints. Calculate the opposition point $X_{i}^{\prime}(t)$ of $X_{i}(t)$.

Step 3. Do flow calculation. Correct the system parameters for the flow calculation. Get the power system operation parameters, determine whether particles meet the bus voltage and generator reactive power and other constraints, modify the cross border values, and calculate their fitness.

Step 4. Compare the fitness of $X_{i}(t)$ and $X_{i}^{\prime}(t)$; select the better one as the current generation.

Step 5. Record the individual optimal solution and the global optimal solution. For each particle, update the individual optimal solution. Then, select the best solution of the individual optimal solutions as the global optimal solution, $t=t+1$.

Step 6. Calculate the current flight speed according to formula (4), and fix the particle position according to formula (1).

Step 7. Select the evolutionary strategies. Go into subroutine (see Figure 1); if $\bmod (t, \delta)=0$, calculate the opposition points as candidate solutions. Else, do crossover and mutation operations and generate candidate solutions according to formula (6) and (7).

Step 8. Update the particle position and modify the cross border values. After reactive compensation and transformer tap variables are discrete, do flow calculation (same as Step 3), compare the fitness of the candidate solution and contemporary individual, and select better one as the next generation.

Step 9. Determine whether it is under terminating condition. If the number of iterations at this time $t$ is less than the maximum number of iterations, go to Step 5, or end the iteration and go to Step 10.

Step 10. Output the optimal solution. Optimal solution includes not only the control strategy of the control variables of each node but also the data of state variables, such as the system voltage of every node, system power loss, and generator reactive power output.

\section{Results and Discussion}

In order to validate the availability of the new oppositionbased improved PSO (OIPSO) in solving power system reactive power optimization problems, IEEE 30 - and IEEE 118-bus systems are employed to be the simulation studies. Two cases are presented in this section. In the first case, run with minimization of real power loss as the objective function and then compare the results with different methods. In the second case, take system network loss, voltage dissatisfaction,
TABLE 1: Comparison of optimal results for different methods.

\begin{tabular}{lcc}
\hline & Parameters & Losses (p.u.) \\
\hline GA [22] & CR $=0.6 ; F=0.01$ & 0.04650 \\
GSA [23] & $G_{0}=100 ; \alpha=10$ & 0.04617 \\
DE [24] & $F=0.2 ; \mathrm{CR}=0.6$ & $0.04550^{*}$ \\
OGSA [25] & $G_{0}=100 ; \alpha=10$ & $0.04498^{*}$ \\
PSO [26] & $w_{\max }=0.9 ; w_{\min }=0.4 ; c_{1}=2$ & 0.04814 \\
CLPSO [26] & $w_{0}=0.9 ; p_{c}=0.4$ & 0.04721 \\
OIPSO & $w_{\max }=0.9 ; w_{\min }=0.4 ; c_{1}=2 ; c_{2 \max }=$ & $\mathbf{0 . 0 4 5 9 4}$ \\
\hline
\end{tabular}

$\mathrm{CR}$ is crossover rate; $F$ is mutation rate; $G_{0}$ is the gravitational constant; $p_{c}$ is learning probability, ${ }^{*}$ The reactive compensation and transformer tap variables are continuous.

and switching operation as the optimization objective and give the optimal settings of control variables.

5.1. IEEE 30-Bus System. IEEE 30-bus system data and operating conditions are given in [21], which has 41 branches, 22 load buses, 6 generators (bus 1 , bus 2 , bus 5 , bus 8 , bus 11 , and bus 13 , while bus 1 is the slack bus and others are PV bus), 4 branches containing four adjustable transformers (branch 9-6, branch 10-6, branch 12-4, and branch 28-27, corresponding to transformers T1, T2, T3, and T4), and 2 shunt capacitors on buses 10 and 24, respectively. In the initial conditions, set the initial generator bus voltages and transformer taps to 1.0 and capacitor values to 0 . The total power loss before optimization is 0.0537 p.u. In this paper, set the population size of particle swarm to be 36 , and the number of iterations is $1000, \delta=2$. In case one, active power loss is studied, and the result is compared with genetic algorithm (GA) [22], gravitational search algorithm (GSA) [23], differential evolution approach (DE) [24], an oppositionbased gravitational search algorithm (OGSA) [25], particle swarm optimization (PSO) [26], and comprehensive learning particle swarm optimization (CLPSO) [26]. Do 50 trials and choose the best one shown in Table 1 .

Although DE [24] and OGSA [25] have obtained the optimal values 0.04550 and 0.4498 , their reactive compensation and transformer tap variables are continuous, which could not satisfy actual operations. The best solution is 0.045936 calculated by the proposed OIPSO algorithm; its average consumption would be $135 \mathrm{~s}$ more than the CPU time of PSO (i.e., $130 \mathrm{~s}$ [26]), due to its discrete control variables and flow calculation of opposition point. And it improves $14.45 \%$, more than $14 \%$ by GSA, and $10.35 \%$ by PSO.

In case two, apply OIPSO to the proposed multiobjective problem, which includes system network loss, voltage dissatisfaction of on-load nodes, and switching operations of adjustable transformer. All expenses involved are shown in Table 2. The results are shown in Table 3.

Table 3 shows the optimal value of the control variables of OIPSO solving reactive power optimization. Comparing with PSO, the OIPSO makes small changes in transformer tap and uses little reactive power compensation to improve voltage quality and increase economic efficiency. Meanwhile, 
TABLE 2: All expenses of reactive power optimization.

\begin{tabular}{lcc}
\hline $\begin{array}{l}\text { Unit network loss } \\
\text { convert expensed } \lambda_{1} \\
(10000 \mathrm{RMB} / \mathrm{MW})\end{array}$ & $\begin{array}{c}\text { Voltage } \\
\text { dissatisfaction } \\
\text { penalty } \lambda_{2} \\
(10000 \mathrm{RMB})\end{array}$ & $\begin{array}{c}\text { Unit operation cost } \\
\text { of transformer tap } \\
\lambda_{3}(10000 \mathrm{RMB})\end{array}$ \\
\hline 69 & 3 & 0.5 \\
\hline
\end{tabular}

TABLE 3: The values of control variables after optimization.

\begin{tabular}{lccc}
\hline Variables & Bus number & PSO [26] & OIPSO \\
\hline$V_{1}$ & 1 & 1.0995 & 1.0995 \\
$V_{2}$ & 2 & 1.0933 & 1.0945 \\
$V_{5}$ & 5 & 1.0697 & 1.0747 \\
$V_{8}$ & 8 & 1.0719 & 1.0764 \\
$V_{11}$ & 11 & 1.0480 & 1.0945 \\
$V_{13}$ & 13 & 1.0945 & 1.0989 \\
$Q_{10}$ & 10 & 0.3000 & 0.2000 \\
$Q_{24}$ & 24 & 0.0500 & 0.0500 \\
$T_{1}$ & $9 \sim 6$ & 0.9375 & 0.9500 \\
$T_{2}$ & $6 \sim 10$ & 1.0000 & 1.0000 \\
$T_{3}$ & $12 \sim 4$ & 1.0125 & 1.0000 \\
$T_{4}$ & $28 \sim 27$ & 0.9750 & 0.9875 \\
$P_{\text {loss }}$ & - & 0.04702 & 0.04625 \\
$S_{V}$ & - & 0.001355 & 0.00573 \\
$S_{T}$ & - & 7 & 5 \\
\hline
\end{tabular}

the results also reflect that satisfactory interval is necessary, if some voltage values exceed the satisfactory interval, but do not go beyond the constraints, the power system could give up a little of satisfactory degree in exchange for active power loss minimum. This situation would not cause security problems; the dissatisfactory penalty could arouse some attention of operators. The satisfactory interval has a function of forecast.

5.2. IEEE 118-Bus System. To test the potential of OIPSO algorithm in solving bigger systems, IEEE 118-bus system is considered, which has 54 generator buses, 64 load buses, 186 transmission lines, 9 transformer taps, and 14 reactive power sources. The system data and operating conditions are given in [26]. The total power loss before optimization is 1.3359 p.u. For case one, Table 4 shows the comparison of results obtained by GSA [23], DE [25], OGSA [25], PSO [26], CLPSO [26], and OIPSO.

OIPSO discovered the best solution, that is, 1.0518 p.u., in which the improvement is $21.27 \%$, more than $4.36 \%$ by GSA, $3.95 \%$ by DE, $4.94 \%$ by OGSA, $1.26 \%$ by PSO, and $2 \%$ by CLPSO. Its success rate lags behind CLPSO by 3 points, and CPU process is 1.196 times as PSO does, but OIPSO has more than 20 times as improvement as CLPSO and PSO. The results in this table indicate the superiority of OIPSO. Comparative PSO- and OIPSO-based convergence profiles of power losses for this test system are presented in Figure 2.
TABLE 4: Comparison of optimal results for different methods.

\begin{tabular}{lccc}
\hline & $P_{\text {loss }}$ (p.u.) & CPU time (s) & Success rate (\%) \\
\hline GSA [23] & 1.2776 & 1199 & $\mathrm{NR}^{*}$ \\
DE [25] & 1.2832 & $\mathrm{NR}^{*}$ & $\mathrm{NR}^{*}$ \\
OGSA [25] & 1.2699 & 1101.3 & $\mathrm{NR}^{*}$ \\
PSO [26] & 1.3191 & 1215 & 59 \\
CLPSO [26] & 1.3096 & 1472 & 73 \\
OIPSO & $\mathbf{1 . 0 5 1 8}$ & 1453 & 70 \\
\hline
\end{tabular}

$\mathrm{NR}^{*}$ : not reported.

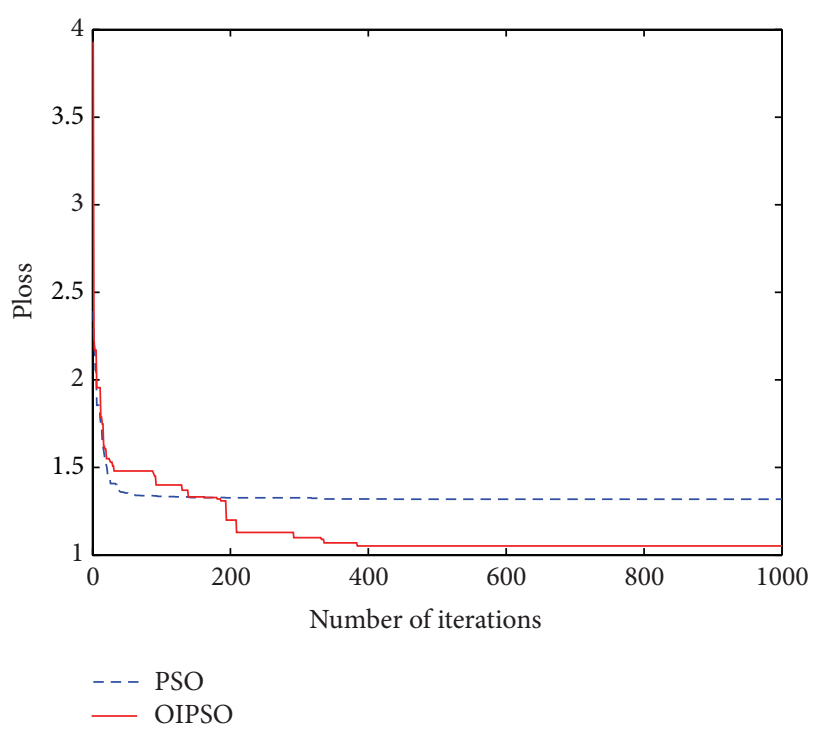

FIGURE 2: Comparative convergence profiles of power loss.

In case two, the multiobjective results of IEEE 118-bus system are given in Table 5; these values of the parameters provide actual operation variables, which is more effective and practical.

\section{Conclusion}

In this paper, we developed an opposition-based improved PSO for multiobjective reactive power optimization. The main novelty of the algorithm is the integration of the opposition-based computing into the basic PSO with inertia weight factors, crossover and mutation, and neighborhood model in order to enhance the diversity and produce some additional exploration ability of the population. The performance of the proposed OIPSO algorithm is demonstrated through its calculation on IEEE 30- and 118-bus systems; the comparison results with other algorithms show that OIPSO has better global search ability and fast searching speed. Meanwhile, when the new multiobjective model of reactive power optimization is being built, switching operation cost is defined by the life cycle cost and is emphasized to reflect the actual situation. So, the optimized results are more realistic and more reliable for practical operation. 
TABLE 5: The values of control variables after optimization.

\begin{tabular}{|c|c|c|c|}
\hline Variables & Bus number & PSO [26] & OIPSO \\
\hline$\overline{V_{1}}$ & 1 & 0.9736 & 1.0844 \\
\hline$V_{4}$ & 4 & 1.0145 & 1.0958 \\
\hline$V_{6}$ & 6 & 0.9969 & 1.0977 \\
\hline$V_{8}$ & 8 & 1.0169 & 1.0956 \\
\hline$V_{10}$ & 10 & 1.0344 & 1.0967 \\
\hline$V_{12}$ & 12 & 0.9868 & 1.0925 \\
\hline$V_{15}$ & 15 & 0.9867 & 1.0899 \\
\hline$V_{18}$ & 18 & 0.985 & 1.0929 \\
\hline$V_{19}$ & 19 & 0.9868 & 1.0893 \\
\hline$V_{24}$ & 24 & 0.9799 & 1.0917 \\
\hline$V_{25}$ & 25 & 1.0014 & 1.0981 \\
\hline$V_{26}$ & 26 & 1.0424 & 1.0955 \\
\hline$V_{27}$ & 27 & 1.002 & 1.0864 \\
\hline$V_{31}$ & 31 & 1.002 & 1.0855 \\
\hline$V_{32}$ & 32 & 1.001 & 1.0866 \\
\hline$V_{34}$ & 34 & 0.9964 & 1.097 \\
\hline$V_{36}$ & 36 & 0.9881 & 1.0932 \\
\hline$V_{40}$ & 40 & 0.9699 & 1.0731 \\
\hline$V_{42}$ & 42 & 1.0282 & 1.088 \\
\hline$V_{46}$ & 46 & 1.0165 & 1.0758 \\
\hline$V_{49}$ & 49 & 1.0213 & 1.0919 \\
\hline$V_{54}$ & 54 & 1.0147 & 1.081 \\
\hline$V_{55}$ & 55 & 1.0113 & 1.0819 \\
\hline$V_{56}$ & 56 & 1.0131 & 1.0814 \\
\hline$V_{59}$ & 59 & 1.0424 & 1.096 \\
\hline$V_{61}$ & 61 & 1.0252 & 1.0911 \\
\hline$V_{62}$ & 62 & 1.0284 & 1.0873 \\
\hline$V_{65}$ & 65 & 1.0377 & 1.0945 \\
\hline$V_{66}$ & 66 & 1.0381 & 1.0983 \\
\hline$V_{69}$ & 69 & 1.0161 & 1.0972 \\
\hline$V_{70}$ & 70 & 0.9551 & 1.0795 \\
\hline$V_{72}$ & 72 & 0.9793 & 1.087 \\
\hline$V_{73}$ & 73 & 0.9269 & 1.0852 \\
\hline$V_{74}$ & 74 & 0.9466 & 1.0593 \\
\hline$V_{76}$ & 76 & 0.938 & 1.0639 \\
\hline$V_{77}$ & 77 & 1.0049 & 1.0777 \\
\hline$V_{80}$ & 80 & 1.0194 & 1.0918 \\
\hline$V_{85}$ & 85 & 1.0303 & 1.095 \\
\hline$V_{87}$ & 87 & 1.09 & 1.0848 \\
\hline$V_{89}$ & 89 & 1.0361 & 1.0979 \\
\hline$V_{90}$ & 90 & 0.9907 & 1.0811 \\
\hline$V_{91}$ & 91 & 1.0087 & 1.0864 \\
\hline$V_{92}$ & 92 & 1.0183 & 1.0965 \\
\hline$V_{99}$ & 99 & 1.0311 & 1.084 \\
\hline$V_{100}$ & 100 & 1.0112 & 1.0975 \\
\hline$V_{103}$ & 103 & 1.0027 & 1.0885 \\
\hline$V_{104}$ & 104 & 1 & 1.091 \\
\hline$V_{105}$ & 105 & 1.0101 & 1.0829 \\
\hline$V_{107}$ & 107 & 0.9857 & 1.0867 \\
\hline
\end{tabular}

TABLE 5: Continued.

\begin{tabular}{|c|c|c|c|}
\hline Variables & Bus number & PSO [26] & OIPSO \\
\hline$V_{110}$ & 110 & 0.9663 & 1.0647 \\
\hline$V_{111}$ & 111 & 0.972 & 1.0728 \\
\hline$V_{112}$ & 112 & 0.9529 & 1.0521 \\
\hline$V_{113}$ & 113 & 1.0241 & 1.0938 \\
\hline$V_{116}$ & 116 & 1.0285 & 1.0979 \\
\hline$Q_{5}$ & 5 & 0 & -0.3 \\
\hline$Q_{34}$ & 34 & 0.1 & 0 \\
\hline$Q_{37}$ & 37 & -0.25 & -0.25 \\
\hline$Q_{44}$ & 44 & 0.1 & 0.05 \\
\hline$Q_{45}$ & 45 & 0.15 & 0.05 \\
\hline$Q_{46}$ & 46 & 0.2 & 0.1 \\
\hline$Q_{48}$ & 48 & 0.05 & 0.1 \\
\hline$Q_{74}$ & 74 & 0.1 & 0.05 \\
\hline$Q_{79}$ & 79 & 0.15 & 0.15 \\
\hline$Q_{82}$ & 82 & 0.2 & 0.2 \\
\hline$Q_{83}$ & 83 & 0.3 & 0 \\
\hline$Q_{105}$ & 105 & 1 & 1.05 \\
\hline$Q_{107}$ & 107 & 0.2 & 0.1 \\
\hline$Q_{110}$ & 110 & 0.05 & 0.05 \\
\hline$T_{1}$ & $5 \sim 8$ & 1.025 & 1.0125 \\
\hline$T_{2}$ & $25 \sim 26$ & 1.0125 & 1.0125 \\
\hline$T_{3}$ & $17 \sim 30$ & 0.9875 & 0.975 \\
\hline$T_{4}$ & $37 \sim 38$ & 0.9875 & 0.9875 \\
\hline$T_{5}$ & $59 \sim 63$ & 1 & 1 \\
\hline$T_{6}$ & $61 \sim 64$ & 1 & 0.9875 \\
\hline$T_{7}$ & $66 \sim 65$ & 1 & 0.9875 \\
\hline$T_{8}$ & $69 \sim 68$ & 0.975 & 1 \\
\hline$T_{9}$ & $80 \sim 81$ & 1.025 & 0.9875 \\
\hline$P_{\text {loss }}$ & - & 1.2033 & 1.1605 \\
\hline$S_{V}$ & - & 0.000585 & 0.007313 \\
\hline$S_{T}$ & - & 18 & 8 \\
\hline
\end{tabular}

\section{Conflict of Interests}

The authors declare that there is no conflict of interests regarding the publication of this paper.

\section{Acknowledgments}

This work was supported by the National Natural Science Foundation of China (Grant no. 51177039), Doctoral Fund of Ministry of Education of China (Grant no. 20090094110011), the Nantong Science and Technology Project, Nantong, China (Grant no. BK2014024), the Changzhou science and technology project, Changzhou, China (Grant no. CJ20130008), and the Open Project of Jiangsu Province Key Laboratory of Environmental Engineering, Nanjing, China (Grant no. KF2014001). 


\section{References}

[1] J. J. Liang, A. K. Qin, P. N. Suganthan, and S. Baskar, "Comprehensive learning particle swarm optimizer for global optimization of multimodal functions," IEEE Transactions on Evolutionary Computation, vol. 10, no. 3, pp. 281-295, 2006.

[2] Y. Shi and R. Eberhart, "Modified particle swarm optimizer," in Proceedings of the IEEE International Conference on Evolutionary Computation, and IEEE World Congress on Computational Intelligence, pp. 69-73, May 1998.

[3] A. Ratnaweera, S. Halgamuge, and H. Watson, "Particle swarm optimization with self-adaptive acceleration coefficients," in Proceedings of the 1st International Conference on Fuzzy Systems and Knowledge Discovery, pp. 246-268, 2003.

[4] S. Burak Akat and V. Gazi, "Particle swarm optimization with dynamic neighborhood topology: three neighborhood strategies and preliminary results," in Proceedings of the IEEE Swarm Intelligence Symposium (SIS '08), pp. 1-8, September 2008.

[5] H. S. Lopes and L. S. Coelho, "Particle swarm optimization with fast local search for the blind traveling salesman problem," in Proceedings of the 5th International Conference on Hybrid Intelligent Systems (HIS '05), pp. 245-250, IEEE, November 2005.

[6] W. Jian and X. Chuanpei, "Study on test generation of sequential circuits based on particle swarm optimization and ant algorithm," in Proceedings of the International Conference on Computer Science and Software Engineering (CSSE '08), pp. 149152, Wuhan, China, December 2008.

[7] X. Wu and G. Huang, "Application of particle filter algorithm in nonlinear constraint optimization problems," in Proceedings of the 8th International Conference on Natural Computation (ICNC '12), pp. 822-826, May 2012.

[8] H. Wang, H. Li, Y. Liu, C. Li, and S. Zeng, "Oppositionbased particle swarm algorithm with Cauchy mutation," in Proceedings of the IEEE Congress on Evolutionary Computation (CEC '07), pp. 4750-4756, Singapore, September 2007.

[9] K. Massimiliano, "A multi-start opposition-based particle swarm optimization algorithm with adaptive velocity for bound constrained global optimization," Journal of Global Optimization, vol. 55, no. 1, pp. 165-188, 2013.

[10] R. Patel, M. M. Raghuwanshi, and L. G. Malik, "Decomposition based multi-objective genetic algorithm (DMOGA) with opposition based learning," in Proceedings of the 4th International Conference on Computational Intelligence and Communication Networks, pp. 605-610, November 2012.

[11] Z. Wu, Z. Ni, C. Zhang, and L. Gu, "Opposition based comprehensive learning particle swarm optimization," in Proceedings of 3rd International Conference on Intelligent System and Knowledge Engineering (ISKE '08), pp. 1013-1019, November 2008.

[12] T. Niknam, M. R. Narimani, R. Azizipanah-Abarghooee, and B. Bahmani-Firouzi, "Multiobjective optimal reactive power dispatch and voltage control: a new opposition-based selfadaptive modified gravitational search algorithm," IEEE Systems Journal, vol. 7, no. 4, pp. 742-753, 2013.

[13] H. Wang, H. Jiang, K. Xu, and G. Li, "Reactive power optimization of power system based on improved particle swarm optimization," in Proceedings of the 4th International Conference on Electric Utility Deregulation and Restructuring and Power Technologies (DRPT '11), pp. 606-609, Shandong, China, July 2011.
[14] R. C. Eberhart and J. Kennedy, "New optimizer using particle swarm theory," in Proceedings of the 6th International Symposium on Micro Machine and Human Science, pp. 39-43, October 1995.

[15] Z. H. Zhan, J. Zhang, Y. Li, and H. S. H. Chung, "Adaptive particle swarm optimization," IEEE Transactions on Systems, Man, and Cybernetics, Part B: Cybernetics, vol. 39, no. 6, pp. 1362-1381, 2009.

[16] S. Rahnamayan, H. R. Tizhoosh, and M. M. A. Salama, "Opposition versus randomness in soft computing techniques," Applied Soft Computing Journal, vol. 8, no. 2, pp. 906-918, 2008.

[17] P. Umapathy, C. Venkataseshaiah, and M. S. Arumugam, "Particle swarm optimization with various inertia weight variants for optimal power flow solution," Discrete Dynamics in Nature and Society, vol. 2010, Article ID 462145, 15 pages, 2010.

[18] S. G. Kim, H. Outhred, and I. Macgill, "Commercialising voltage regulation in nodal electricity spot markets," International Energy Journal, vol. 6, no. 1, pp. 219-228, 2005.

[19] Y. Song and J. Li, "Analysis of the life cycle cost and intelligent investment benefit of smart substation," in Proceedings of the IEEE Innovative Smart Grid Technologies-Asia (ISGT-Asia '12), pp. 1-5, IEEE, Tianjin, China, May 2012.

[20] X. Jiang, W. Xuan, and X. Chen, "Research and application of power transformer life cycle cost model," Journal of Fuzhou University (Natural Science Edition), vol. 40, no. 3, pp. 357-361, 2012 (Chinese).

[21] R. Li and S.-Q. Sheng, "An improved particle swarm optimization algorithm for reactive power optimization," in Asia-Pacific Power and Energy Engineering Conference (APPEEC '11), pp. 1-5, March 2011.

[22] S. Durairaj, P. S. Kannan, and D. Devaraj, "Application of genetic algorithm to optimal reactive power dispatch including voltage stability constraint," Journal of Energy \& Environment, pp. 6373, 2005.

[23] S. Duman, Y. Sonmez, U. Guvenc, and N. Yorukeren, "Application of gravitational search algorithm for optimal reactive power dispatch problem," in Proceedings of the International Symposium on INnovations in Intelligent SysTems and Applications (INISTA '11), pp. 519-523, June 2011.

[24] A. A. A. E. Ela, M. A. Abido, and S. R. Spea, "Differential evolution algorithm for optimal reactive power dispatch," Electric Power Systems Research, vol. 81, no. 2, pp. 458-464, 2011.

[25] B. Shaw, V. Mukherjee, and S. P. Ghoshal, "Solution of reactive power dispatch of power systems by an opposition-based gravitational search algorithm," International Journal of Electrical Power and Energy Systems, vol. 55, pp. 29-40, 2014.

[26] K. Mahadevan and P. S. Kannan, "Comprehensive learning particle swarm optimization for reactive power dispatch," Applied Soft Computing Journal, vol. 10, no. 2, pp. 641-652, 2010. 


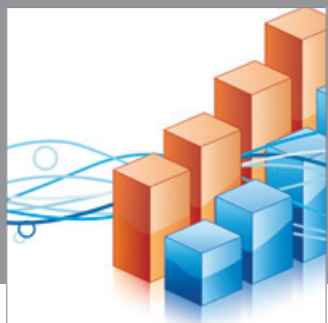

Advances in

Operations Research

mansans

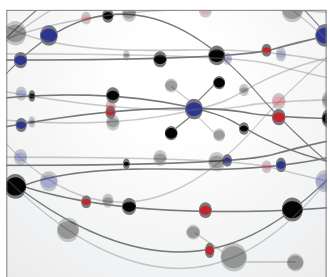

The Scientific World Journal
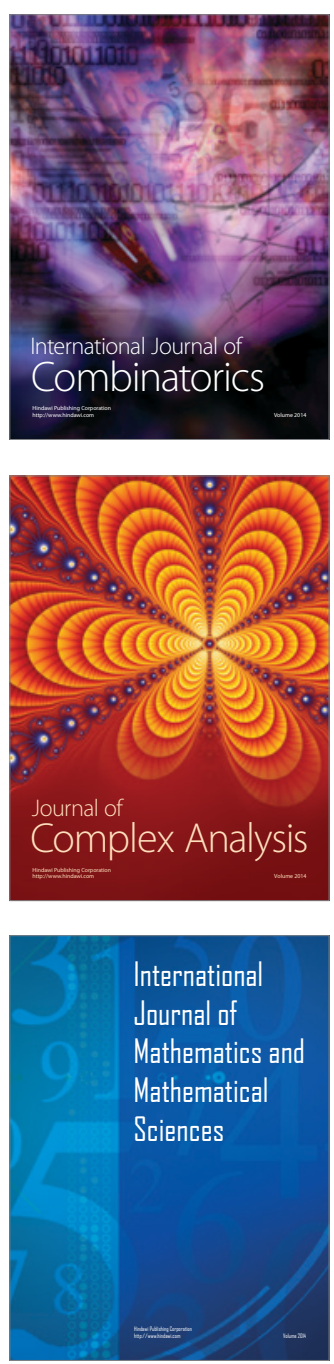
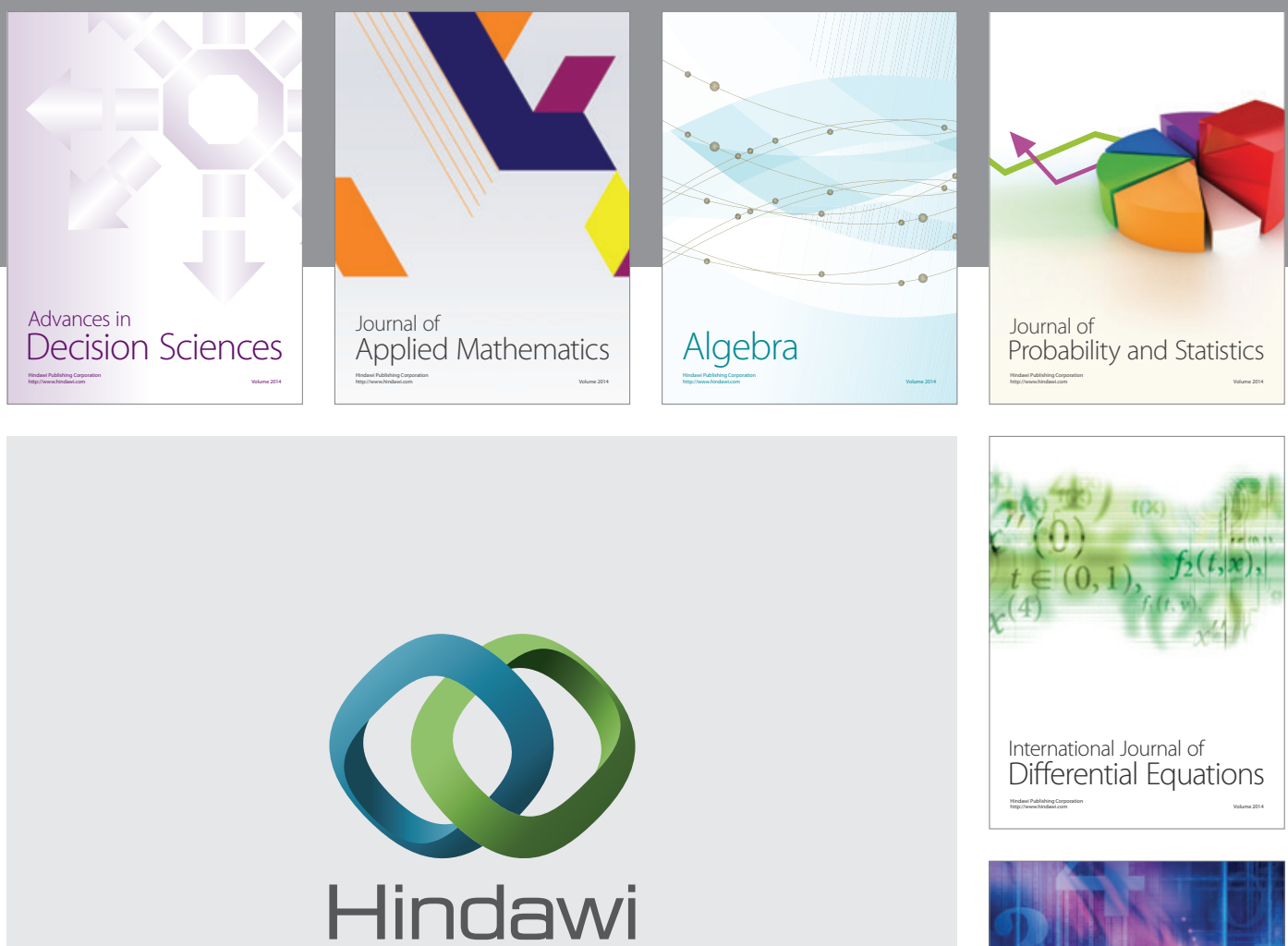

Submit your manuscripts at http://www.hindawi.com
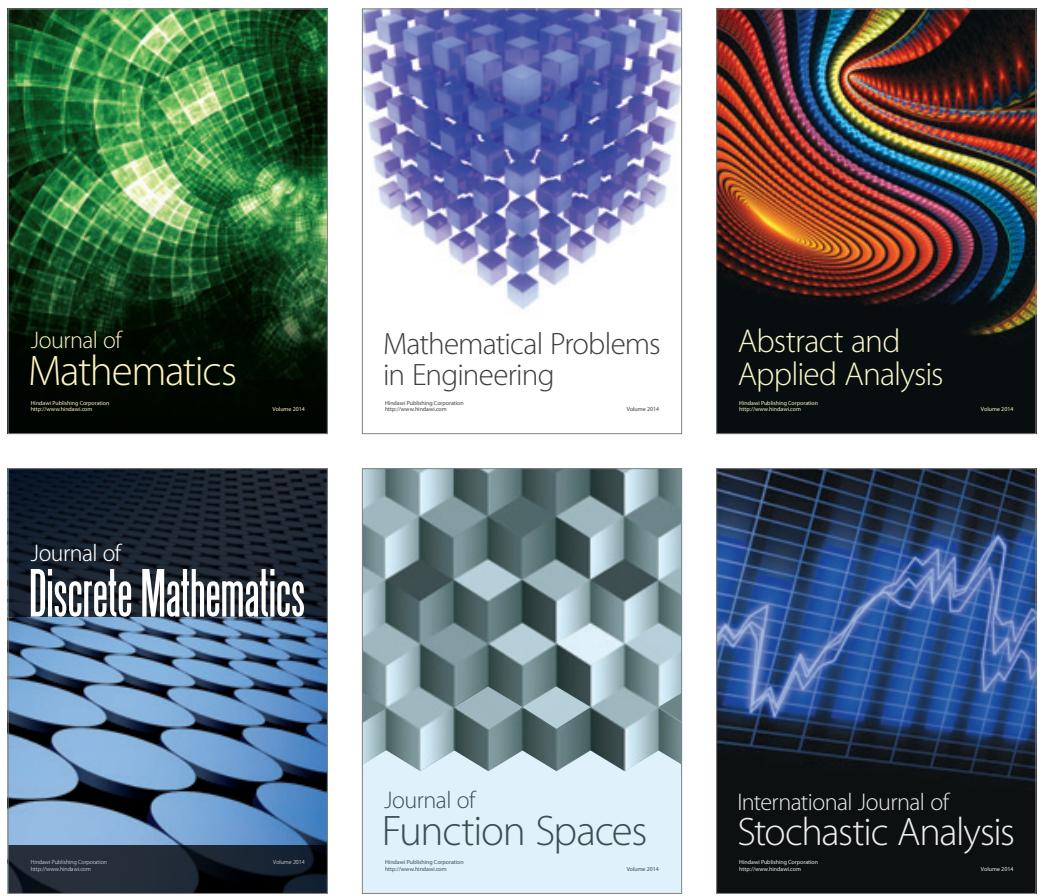

Journal of

Function Spaces

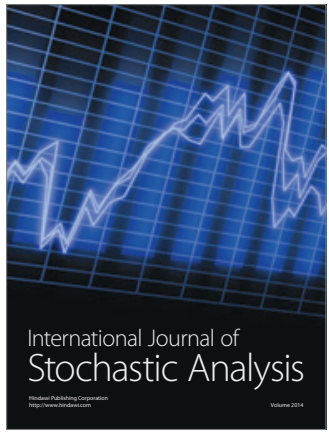

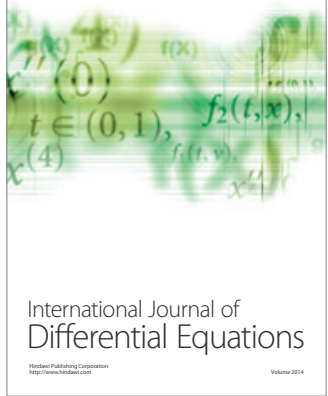
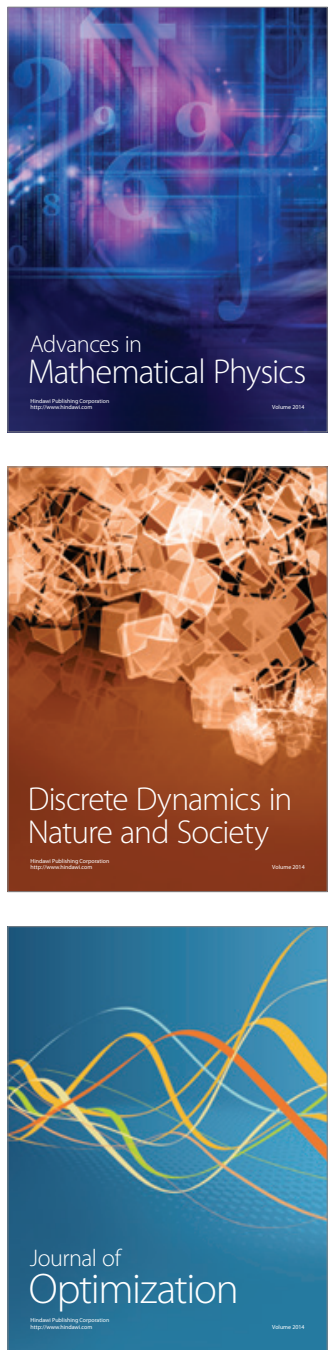